\title{
Alterations in Plasma miR-21, miR-590, miR-192 and miR-215 in Patients with Idiopathic Pulmonary Fibrosis and Their Clinical Importance
}

Hulya Dirol ( $\boldsymbol{\sim}$ hulyadirol@akdeniz.edu.tr)

Akdeniz University Faculty of Medicine Chest Department https://orcid.org/0000-0002-7712-6467

Aslı Toylu

Akdeniz University Medical Genetics

Aliye Candan Ogus

Akdeniz University Faculty of Medicine Chest Department

Aykut Cilli

Akdeniz University Faculty of Medicine Chest Department

Omer Ozbudak

Akdeniz University Faculty of Medicine Chest Department

Ozden Altıok Clark

Akdeniz University Medical Genetics

Tulay Ozdemir

Akdeniz University Faculty of Medicine Chest Department

\section{Research Article}

Keywords: Idiopathic Pulmonary Fibrosis, miR-21, miR-590, miR-192, and miR-215

Posted Date: June 1st, 2021

DOl: https://doi.org/10.21203/rs.3.rs-471899/v1

License: (c) (i) This work is licensed under a Creative Commons Attribution 4.0 International License.

Read Full License 


\section{Abstract}

Background: It is well-established that microRNAs (miRNAs) have regulatory roles in the fibrotic processes of various tissues. Studies revealed that some miRNAs play a pivotal role in the development of idiopathic pulmonary fibrosis (IPF).

Methods: In this single-center, cross-sectional study, the plasma levels of miR-21, miR-590, miR-192, and miR-215 in IPF ( $n=88)$ and the control $(n=20)$ group were investigated using real-time PCR. The pulmonary function tests and the plasma sampling of participants were carried out simultaneously. Patients were grouped according to age, forced vital capacity, diffusing capacity for carbon monoxide (DLCO), and the Gender-Age-pulmonary Physiology (GAP) score. The expression levels of the target miRNAs between these clinical subgroups were compared.

Results: The IPF and control groups were similar in terms of gender and age. The mean plasma miR-21 and miR-590 levels in IPF were significantly higher than in the control ( $p<0.0001, p<0.0001$, respectively). There was no significant difference in miR-192 and miR-215 levels between the groups. While miR-21 and miR-590 correlated positively with age $(p=0.041, p=0.007$, respectively), miR-192 and miR-215 displayed a negative correlation with age $(p=0.0002, p<0.0001$, respectively). MiR-192 and miR-215 increased as the GAP score decreased and miR-192 level in patients with honeycombing was significantly lower than in those without honeycombing $(p=0.003)$.

Conclusions: Our results suggest that higher plasma levels of miR-21 and miR-590 were related to the presence of IPF. While all target miRNAs correlated with age, the miR-21 and miR-590 were associated with DLCO, and miR-192 and miR-215 were associated with GAP score and honeycombing.

\section{Background}

Idiopathic pulmonary fibrosis (IPF) is a progressive fatal fibrotic lung disease with an unknown etiology. Although, myofibroblasts related with the excessive production of extracellular matrix appear to play a central role in the pathogenesis of this disease, the cells, and pathways involved in the pathogenesis have not been fully elucidated [1].

MicroRNA (miRNA) molecules are small non-coding endogenous RNAs that regulate the translation of their target messenger RNAs [2]. They have a modulatory role in various cells and in almost every cellular function. Also, majority of the fibrotic processes are currently thought to be regulated by miRNAs in fibrotic diseases [3].

According to recent evidence, some miRNAs which have a central role in the fibrotic signaling pathways, are involved in the development of IPF also [4]. Studies have shown that expression of miR-21 is increased in lung tissue of patients with IPF and activity of lung fibroblasts is promoted with miR-21 overexpression [5]. miR-21 was also found in the serum of IPF patients [6]. Moreover, miR-21 has been associated with the diagnosis, progression, and severity of IPF [7]. 
Interestingly, another miRNA molecule, miR-590, has a similar nucleotide sequence to miR-21 [8]. Therefore, it is predicted that these two miRNA molecules could share target mRNA molecules and take part in similar cellular pathways. miR-21 has been shown to contribute to both pulmonary and cardiac fibrosis processes [9]. Although miR-590 has been reported to be associated with cardiac fibrosis, its role in pulmonary fibrosis has not been studied yet [10].

The miR-192 molecule has also been reported to be involved in the development of fibrosis. Extensive studies are being conducted on the regulatory role of miR-192 in fibrosis, particularly in renal fibrosis. Furthermore, miR-192 was shown to be increased in bleomycin-induced fibrotic lungs of mice, and miR192 was found in the sputum of patients with IPF [11, 12]. However, there is no data about its relation with IPF severity. As between miR-21 and miR-590, there is a strong sequence homology between miR192 and miR-215 molecules. Although miR-215 was reported to regulate fibroblast function, there is no data about its role in lung fibrosis [13].

Currently, the interest in the association between circulating extracellular miRNA and the development and prognosis of IPF has been increasing. Therefore, the plasma expression levels of miR-21, miR-590, miR-192, and miR-215 molecules in IPF and their relationship with the clinical features were investigated in this study.

\section{Methods}

\section{Study Design and Participants}

This single-center, cross-sectional study was conducted in the Chest Diseases Department of Akdeniz University Hospital between June 2018 and August 2020. IPF patients who are meeting the criteria of the 2018 American Thoracic Society / European Respiratory Society Clinical Practice Guidelines, and do not have heart disease, cancer, renal failure, or hepatic disease, were included in the IPF group [9]. The control group consisted of healthy subjects who matched the IPF group in terms of age and gender.

\section{Clinical Features}

The demographic data and clinical characteristics of the patients (age, gender, smoking history, symptoms, drug therapy, lung biopsy) were obtained. Recent high-resolution computer tomography (HRCT) images of subjects were used and subpleural, basal dominant reticulation with honeycombing was accepted as usual interstitial pneumonia (UIP) pattern. The severity of dyspnea was determined with a modified Medical Research Council (mMRC) Questionnaire. The gender-age-pulmonary physiology (GAP) score was calculated using gender, age, \% of predicted forced vital capacity (FVC), and \% of predicted diffusing capacity for carbon monoxide (DLCO). Spirometry and helium diffusion tests were applied to evaluate respiratory functions in the IPF group and simultaneously blood samples were taken for miRNA analysis. The data of age, gender, smoking history, and comorbidities were obtained from 
healthy control subjects. Blood samples were collected for miRNA analysis but spirometry and helium diffusion tests were not performed in the control group.

Patients were grouped according to age, FVC (\% of predicted), DLCO (\% of predicted), and GAP score. The age groups were $<65$ years (younger group) and $\geq 65$ years (older group). Three subgroups were formed based on FVC values $(<65 \%, 65-80 \%,>80 \%)$, DLCO values $(<45 \%, 45-70 \%,>70 \%)$, and GAP scores $(0-2,3-4$, $5-8)$.

\section{miRNA Expression Analysis}

Expression levels of target miRNA molecules were examined with real-time PCR. Plasma was obtained from peripheral blood samples and stored at $-80^{\circ} \mathrm{C}$. miRNA was isolated from plasma with microRNA Purification Kit (Norgen Biotek Corp., Canada) and cDNA was synthesized using miRCURY LNA RT Kit (Qiagen, USA). Real-time PCR was performed with hsa-miR-21-5p, hsa-miR-590-5p, has-miR-192-5p, hasmiR-215-5p, and hsa-miR-16-5p miRCURY LNA miRNA PCR Assay reagents with miRCURY LNA SYBR Green PCR Kit (Qiagen, USA) on LightCycler 480 II instrument via LightCycler 4801.5 software (Roche Diagnostics $\mathrm{GmbH}$, Germany). Relative quantification analysis of target miRNA genes was performed with REST-MCS software by using miR-16 as a reference gene [14].

\section{Human ethics}

Written informed consent was obtained from all patients and control subjects. Approval for study was granted by the Clinical Research Ethics Committee of Akdeniz University Faculty of Medicine on 17.01.2018 (decision no: 53, dated: 17.01.2018). The study was supported by Akdeniz University Scientific Research Projects Coordination Unit (Project Number: TTU-2019-4793).

\section{Statistical Analysis}

Statistical analysis of the data was performed using GraphPad Prizm 9 (GraphPad Software, USA) program. Descriptive statistics for categorical variables were reported as number $(n)$ and percentage (\%) and for continuous variables as mean \pm standard deviation (SD) or mean \pm standard error of mean (SEM) values. Differences of means between two independent groups were examined with the Mann-Whitney $U$ test, and between more than two independent groups with Kruskal Wallis analysis. Relationships of two independent categorical variables were interpreted by Chi-Square analysis or with Fisher's Exact Test. The correlation of independent continuous variables was analyzed with Spearman's rank correlation test. In all analyses, a value of $p \leq 0.05$ was considered statistically significant.

\section{Results}




\section{Demographic features, respiratory functions, and radiological findings}

Eighty-eight patients with IPF and 20 healthy control subjects were evaluated in this study. IPF group comprised $82(93.18 \%)$ males and the mean age was $66.6 \pm 8.6$ years [Table 1]. There was no difference between IPF and control group in terms of gender, age, and smoking amount but the ratio of smokers was higher in IPF than in control group ( $71 \%$ vs. $45 \%, p=0.038)$. Majority of the patients $(81.8 \%)$ had dyspnea in various degrees and the median mMRC score was 2 . The mean FVC (\% of pred.) was $71.3 \pm 17.1 \%$ and DLCO (\% of pred.) was $60.6 \pm 17.8 \%$.

Table 1

Demographic features, respiratory functions and radiological findings of participants.

\begin{tabular}{|c|c|c|}
\hline \multirow[t]{2}{*}{ Characteristic } & \multirow{2}{*}{$\begin{array}{l}\text { Control } \\
n=20\end{array}$} & \multirow{2}{*}{$\begin{array}{l}\text { IPF } \\
n=88\end{array}$} \\
\hline & & \\
\hline Age, mean \pm SD & $63 \pm 7.2$ & $66.6 \pm 8.6$ \\
\hline Gender (Male) & $80 \%$ & $82 \%$ \\
\hline Smoking history & $45 \%$ & $71 \% *$ \\
\hline Smoking amount $(\mathrm{P} / \mathrm{Y})$, mean $\pm \mathrm{SD}$ & $31.8 \pm 30.3$ & $31.8 \pm 18.3$ \\
\hline Dyspnea & & $81.8 \%$ \\
\hline FVC (\%pred.), mean \pm SD & - & $71.3 \pm 17.1$ \\
\hline DLCO (\%pred.) $(n=82)$, mean \pm SD & - & $60.6 \pm 17.8$ \\
\hline GAP score, median (min-max) & - & $3(0-8)$ \\
\hline HRCT (UIP pattern) ( $n=84)$ & - & $76 \%$ \\
\hline Drug therapy $(n=66)$ & & $75 \%$ \\
\hline \multicolumn{3}{|c|}{$\begin{array}{l}\text { (IPF: Idiopathic pulmonary fibrosis, P/Y: Packet/Year, SD: Standard Deviation, FVC: Forced Vital } \\
\text { Capacity, \% pred.: \% of predicted, DLCO: Diffusing Lung capacity for Carbon monoxide, GAP: Gender- } \\
\text { Age-pulmonary Physiology, min: Minimum, max: Maximum, HRCT: High-Resolution Computed } \\
\text { Tomography, UIP: Usual Interstitial Pneumonia, n: Number, }{ }^{\star} p=0.038 \text {, smoking rate in IPF versus } \\
\text { control with Fisher's Exact test) }\end{array}$} \\
\hline
\end{tabular}

Sixty-four (72.7\%) of the IPF patients had a UIP pattern and 24 (27.3\%) patients had a surgical biopsy for the diagnosis. The mean age of patients with honeycombing on HRCT was significantly higher than that of patients without honeycombing (68.5 vs. 62.1 years, $p=0.0027$ ). The median GAP score was 3 and antifibrotic drugs were being taken by $75 \%$ of the patients and majority $(74 \%)$ of these drugs were pirfenidone.

Clinical features of IPF patients were different between younger and older groups [Table 2]. Gender and smoking features in IPF were similar in both age-groups. UIP pattern was significantly more common $(p=$ 
$0.015)$ and the GAP score was higher $(p=0.001)$ in the older group however, the mean FVC and DLCO values were similar.

Table 2

The effect of age on demographic features, respiratory functions and radiological findings of IPF patients.

\begin{tabular}{|c|c|c|c|}
\hline \multirow[t]{2}{*}{ Characteristic } & Age $<65$ & Age $\geq 65$ & \multirow[t]{2}{*}{$p$} \\
\hline & $n=34$ & $n=54$ & \\
\hline Gender (Male) & $80 \%$ & $84 \%$ & Ns \\
\hline Smoking history & $85 \%$ & $68 \%$ & Ns \\
\hline FVC (\%pred), mean \pm SD & $67.0 \pm 17.6$ & $74.1 \pm 16.3$ & Ns \\
\hline DLCO (\%pred) $(n=82)$, mean \pm SD & $60.5 \pm 19.3$ & $60.7 \pm 16.9$ & Ns \\
\hline GAP score, median (min-max) & $2(0-7)$ & $4(2-8)$ & $p=0.001^{*}$ \\
\hline HRCT (UIP pattern) & $60 \%$ & $85 \%$ & $p=0.015^{\star \star}$ \\
\hline Drug therapy & $26.5 \%$ & $24 \%$ & Ns \\
\hline \multicolumn{4}{|c|}{$\begin{array}{l}\text { (IPF: Idiopathic pulmonary fibrosis, FVC: Forced Vital Capacity, \% pred.: \% of predicted, SD: Standard } \\
\text { Deviation, DLCO: Diffusing Lung capacity for Carbon monoxide, GAP: Gender-Age-pulmonary } \\
\text { Physiology, min: Minimum, max: Maximum, HRCT: High-Resolution Computed Tomography, UIP: } \\
\text { Usual Interstitial Pneumonia, n: Number, Ns: Not significant, *With Chi-Square Analysis, **With Fisher's } \\
\text { Exact Test) }\end{array}$} \\
\hline
\end{tabular}

\section{miRNA levels in IPF and control group}

The mean plasma miR-21 and miR-590 levels in IPF were at least two-fold higher than in control group [Figure 1]. There was no significant difference in the mean plasma miR-192 and miR-215 levels between the groups. Also, plasma levels of target miRNA molecules weren't altered significantly with smoking in both the control (miR-21: $0.65 \pm 0.35$ vs. $0.77 \pm 0.64, p>0.05$; miR-590: $0.52 \pm 0.31$ vs. $0.63 \pm 0.51, p>0.05$; miR-192: $1.50 \pm 0.85$ vs. $1.31 \pm 0.45, p>0.05$; miR-215: $1.60 \pm 0.86$ vs. $1.60 \pm 0.71, p>0.05$; smoking history no vs. yes, respectively) and IPF groups (miR-21: $1.49 \pm 1.13$ vs. $1.43 \pm 0.90, p>0.05$; miR-590: $1.31 \pm 0.90$ vs. $1.41 \pm 1.08, p>0.05$; miR-192: $1.29 \pm 0.58$ vs. $1.33 \pm 0.40, p>0.05$; miR-215: $1.35 \pm 0.57$ vs. $1.48 \pm 0.69, p>0.05$; smoking history no vs. yes respectively). Additionally, the mean target miRNA levels were not altered according to anti-fibrotic drugs (miR-21: $1.33 \pm 0.78$ vs. $1.48 \pm 1.03, p>0.05$; miR-590: $1.20 \pm 0.97$ vs. $1.44 \pm 1.04, p>0.05$; miR-192: $1.35 \pm 0.55$ vs. $1.31 \pm 0.42, p>0.05$; miR-215: $1.46 \pm 0.71$ vs. $1.44 \pm 0.65, p>0.05$; anti-fibrotic therapy no vs. yes, respectively). A positive correlation was found between the levels of miR-21 and miR-590 and between the levels of miR-192 and miR-215 in both IPF and control groups [Figure 2].

\section{The effect of age on serum miRNA levels}


In the IPF group, there was a positive correlation between age and both miR-21 and miR-590, while miR192 and miR-215 were negatively correlated with age [Figure 3]. The mean miR-21 and miR-590 levels were significantly higher in the older group than in the younger group $(1.1 \pm 0.6$ vs. $1.6 \pm 1.0, p=0.032,1.0$ \pm 0.5 vs. $1.6 \pm 1.2, p=0.039$, respectively). miR-192 and miR-215 levels were significantly lower in older group than in younger group $(1.5 \pm 0.5$ vs. $1.2 \pm 0.3, p=0.004,1.6 \pm 0.6$ vs. $1.3 \pm 0.6, p=0.001$, respectively).

In the control group, none of the miRNA molecules correlated with age [Figure 3]. When the control subjects were separated into younger $(n=12)$ and older $(n=8)$ age groups, although miR-21 and miR-590 levels were similar, both miR-192 and miR-215 levels were significantly higher in the older group than in the younger group (miR-192: $1.2 \pm 0.7$ vs. $1.6 \pm 0.5, p=0.047$, miR-215: $1.3 \pm 0.7$ vs. $1.9 \pm 0.6, p=0.031$ ).

\section{Association of miRNAs with pulmonary function tests, GAP score and radiological findings in patients with IPF}

In FVC subgroups, no difference was determined in plasma levels of miR-590, miR-192, and miR-215 [Supplemental Table-1], but a slight difference was seen in miR-21 level. miR-21 level of patients with $\mathrm{FVC}>80 \%$ was higher than that of patients with FVC $66-80 \%(1.6 \pm 0.9$ vs. $1.2 \pm 0.9, p=0.012)$.

In DLCO subgroups, there was no significant difference in miR-192 and miR-215 levels [Supplemental Table-1]. Both miR-21 and miR-590 were reduced in patients with DLCO $<45 \%$ compared to other DLCO subgroups, but the miR-21 and miR-590 levels of this group were similar to those of control subjects [Figure 4]. Although plasma miR-21 and miR-590 levels weren't significantly changed among DLCO subgroups (with Kruskal Wallis test), patients with the highest DLCO levels showed significantly increased miR-590 levels compared to patients with the lowest DLCO values (1.5 \pm 0.9 vs. $1.0 \pm 1.1, p=$ 0.022 with Mann-Whitney U test).

miR-21 and miR-590 did not change according to GAP score [Supplemental Table-2]. In contrast, miR-192 and miR-215 were reduced along with the increased GAP scores [Figure 5]. The plasma miR-192 level was significantly reduced in the patients whose GAP scores are higher than $2(p=0.010)$. Although the plasma miR-215 levels were not significantly changed with GAP score $(p=0.071)$, their levels were reduced in the patients with a GAP score higher than 2 ( $p<0.05$ with Mann Whitney $U$ test).

Related to the radiological findings, the mean plasma miR-215 levels were lower in patients with honeycombing compared to those without honeycombing, but the difference was not significant [Supplemental Table-2]. The mean plasma miR-192 level in patients with honeycombing was significantly lower than in those without honeycombing $(p=0.003)$ [Figure 6].

\section{Discussion}


In this study, we investigated plasma miR-21, miR-590, miR-192, and miR-215 levels in IPF patients. The plasma miR-21 and miR-590 levels in IPF group were significantly higher than those of the control group, but there was no significant difference in miR-192 and miR-215 levels. Moreover, miR-21 and miR-590 were associated with age and DLCO, while miR-192 and miR-215 were associated with age, GAP score, and honeycombing. These findings suggested that miR-21 and miR-590 could take a role in the development of IPF, additionally, miR-192 and miR-215 also could be related to the progression of the IPF.

MiRNA molecules have been reported to be involved in lung fibrosis. Formerly, the expression level of miR21 was found to increase both in the lung tissue of bleomycin-treated mice and in the lungs of IPF patients [5]. Then, the serum miR-21 was suggested as a representative of miR-21 expression in the lung tissue of IPF patients [15], and additionally, increased serum miR-21 levels were reported in two different IPF studies [7, 16]. In our study, the plasma miR-21 levels were significantly higher in IPF than in the control. Together with former studies, our results provide further evidence that miR-21 levels are elevated in plasma of the patients with IPF.

To the best of our knowledge, there is no data about the plasma levels of miR-590, miR-192, and miR-215 in IPF. Each one of these miRNAs has been reported to be involved in various fibrotic diseases but only miR-192 was associated with lung fibrosis $[10,17,18]$. MiR-192 expression was found to be increased in the bleomycin-induced fibrotic lungs of mice and in the sputum of IPF patients [11, 12]. Additionally, miR192 has been shown to be a modulator of airway remodeling in patients with asthma [13]. In this study, the plasma miR-590 levels were significantly higher in IPF than in the control, while the expression levels of both miR-192 and miR-215 were similar.

Besides the presence of IPF, our study provided evidence that plasma miRNAs could display expressional alterations with the severity of IPF. The mean plasma level of miR-590 was high in IPF group, but it was decreased as the DLCO values decreased. This data suggests that plasma miR- 590 expression increases with IPF development, but along with the disease progression it reduces. Thus, the suppression of miR590 expression could be related to a negative feedback mechanism associated with the continuing fibrotic processes. Moreover, plasma level of miR-192 did not change by the presence of IPF, but it was decreased as the radiological severity or the GAP score increased. So, miR-192 could be a promising plasma biomarker for the prognosis of IPF.

In this study, miR-21 was positively correlated with miR-590 in both control and IPF groups. Previously, they were observed to display an inverse expression pattern in ulcerative colitis and there was no correlation between them [19]. Furthermore, our results showed that both miR-21 and miR-590 positively correlated with age in IPF group. The circulating levels of miR-21 were previously reported to increase with age and the miR-590 levels were also found elevated in older subjects [20]. Supporting these data, miR-21 was reported as DNA damage-related circulatory biomarker for aging [21]. Interestingly, we observed that miR-192 and miR-215 were negatively correlated with age in IPF group. Although circulating miR-192 levels were suggested to decrease with age, miR-21 and miR-192 were found elevated in circulating vesicles of aged mice [22]. Since miRNA molecules in the circulation are distributed in 
several compartments as protein-bound or in exosomes, currently it's unknown whether these different compartments are particularly associated with the specific diseases [23]. Importantly, the effect of age on miRNA levels seems to be depending on the severity of the current disease and the specific compartment of miRNAs in which they are distributed.

The interaction of miRNAs with fibrotic processes could be associated with the fibrotic signaling pathways. TGFb molecule, closely related to miR-21, is an important factor in pulmonary fibrosis. In renal fibrosis, TGFb induces fibrosis with Smad3 directed upregulation of miR-21, while miR-21 modulates TGFb signaling by reducing the inhibitory Smad7 molecule. Lung injury stimulates miR-21 and TGFb expression and also inhibits Smad7 expression [24]. During bronchial airway remodeling, TGFb induces miR-21 expression but then miR-21 inhibits Smad7 and further stimulates TGFb signaling [25]. Collectively, miR-21 seems to be a positive feedback regulator of TGFb signaling in fibrosis development.

Together with miR-21, miR-590 has also been implicated in the fibrotic TGFb signaling. Although TGFb reduces miR-590 expression, miR-590 directly inhibits both TGFb and TGFb receptors to suppress its signaling pathway, but miR-590 also inhibits Smad3 and Smad7 expressions [26-28]. Therefore, it has been suggested that miR-590 and TGFb molecules act as negative regulators of each other. However, these data are in contrast with our observation of a higher plasma level of miR-590 in IPF patients than in healthy subjects. A possible explanation for this discrepancy might be associated with the inflammatory response. Pro-inflammatory cytokines regulate miR-590 expression by JAK/STAT pathway [29]. During inflammation processes, STAT3 activates STAT5, and STAT5 is reported to increase miR-590 expression, whereas STAT3 is identified as a target of miR-590. In addition, STAT3/5 proteins reported to be involved in the regulation of EMT with TGFb signaling in pulmonary fibrosis [30]. These data suggest that miR-590 might have a dual role in both inflammation and fibrosis during IPF progression as a negative feedback regulator.

Interestingly, TGFb-induced miR-192 functions are much more heterogeneous, miR-192 can inhibit or stimulate fibrosis depending on the cell type. TGFb signaling with Smad3 promotes miR-192 expression, whereas Smad7 reduces miR-192 expression. Previously, miR-192 expression was found reduced in the cigarette smoke-exposed lung tissue. However, during bleomycin-induced pulmonary fibrosis, miR-192 expression was increased at first but reduced later [11]. Also, high levels of exosomal miR-192 were found in the sputum samples of IPF patients [12]. Altogether these data support that the effect of TGFb on fibrosis via miR-192 differs according to the target cell type and miR-192 may play a regulatory role in IPF disease development and progression.

There are limitations in this study. The control group was small in number and the correlation of target miRNAs with age in control group needs to be analyzed with further studies. In addition, it's not possible to know whether miRNAs in the plasma of IPF patients are originated from lung tissues or extrapulmonary tissues, or circulating cells.

In conclusion, the plasma miR-590 and miR-21 levels were found significantly higher in the IPF group than in the control group. Moreover, the miR-21 and miR-590 levels were found to be related to age and 
DLCO, while miR-192 and miR-215 levels were associated with age, GAP score, and honeycombing.

Therefore, these miRNAs may take a regulatory role in the development and progression of IPF and may be utilized as a diagnostic or prognostic tool or potentially, as a new therapeutic target. Nevertheless, further research is needed to better understand the roles of plasma miRNAs in IPF.

\section{Declarations}

\section{Funding}

This work was supported by a grant from Akdeniz University Scientific Research Projects Coordination Unit (Project Number: TTU-2019-4793).

\section{Conflicts of interest}

The authors declare that they have no conflict of interest.

\section{Ethics approval}

This work was approved by the Clinical Research Ethics Committee of Akdeniz University Faculty of Medicine on 17.01.2018 (decision no: 53, dated: 17.01.2018).

\section{Consent to Participate}

Written informed consent was obtained from all patients and control subjects.

\section{Consent to Publish}

All of the authors agree to submit the manuscript to Molecular Biology Reports for publication.

\section{Authors' contributions}

$\mathrm{HD}, \mathrm{AT}, \mathrm{ACO}, \mathrm{AC}, \mathrm{OO}, \mathrm{OAC}$ and $\mathrm{TO}$ designed the experiments and proofread the manuscript. HD and AT performed the experiments and analyzed the data. HD and AT also wrote the manuscript. HD, ACO, AC, O०, TO helped in providing blood samples.

\section{Availability of data and material}

All data and materials of the work are available behind the corresponding author. 


\section{References}

1. Selman M, King TE, Pardo A. American Thoracic Society; European Respiratory Society; American College of Chest Physicians. Idiopathic pulmonary fibrosis: prevailing and evolving hypotheses about its pathogenesis and implications for therapy. Ann Intern Med. 2001;134(2):136-51.

2. he L, haNNoN Gj. MicroRNAs: small RNAs with a big role in gene regulation. Nat Rev Genet 2004;5:522-531.

3. Bowen T, Jenkins RH, Fraser DJ. MicroRNAs, transforming growth factor beta-1, and tissue fibrosis. J Pathol. 2013;229(2):274-85.

4. Martinez FJ, Collard HR, Pardo A, et al. Idiopathic pulmonary fibrosis. Nat Rev Dis Primers. 2017;3:17074.

5. Liu G, Friggeri A, Yang Y, et al. miR-21 mediates fibrogenic activation of pulmonary fibroblasts and lung fibrosis. J Exp Med. 2010;207(8):1589-97.

6. Tsitoura E, Wells AU, Karagiannis K, et al. MiR-185/AKT and miR-29a/collagen 1a pathways are activated in IPF BAL cells. Oncotarget. 2016;7(46):74569-74581.

7. Makiguchi T, Yamada M, Yoshioka Y, et al. Serum extracellular vesicular miR-21-5p is a predictor of the prognosis in idiopathic pulmonary fibrosis. Respir Res. 2016;17(1):110.

8. Stolzenburg LR, Harris A. The role of microRNAs in chronic respiratory disease: recent insights. Biol Chem. 2018;399(3):219-234.

9. Raghu G, Remy-Jardin M, Myers JL, et al. American Thoracic Society, European Respiratory Society, Japanese Respiratory Society, and Latin American Thoracic Society. Diagnosis of Idiopathic Pulmonary Fibrosis. An Official ATS/ERS/JRS/ALAT Clinical Practice Guideline. Am J Respir Crit Care Med. 2018;198(5):e44-e68.

10. Yuan X, Pan J, Wen L, et al. MiR-590-3p regulates proliferation, migration and collagen synthesis of cardiac fibroblast by targeting ZEB1. J Cell Mol Med. 2020 ;24(1):227-237.

11. Cushing L, Kuang PP, Qian J, et al. miR-29 is a major regulator of genes associated with pulmonary fibrosis. Am J Respir Cell Mol Biol. 2011;45(2):287-94.

12. Njock MS, Guiot J, Henket MA, et al. Sputum exosomes: promising biomarkers for idiopathic pulmonary fibrosis. Thorax. 2019;74(3):309-312.

13. Lou L, Tian M, Chang J, et al. MiRNA-192-5p attenuates airway remodeling and autophagy in asthma by targeting MMP-16 and ATG7. Biomed Pharmacother. 2020;122:109692.

14. Pfaffl MW, Horgan GW, Dempfle L. Relative expression software tool (REST) for group-wise comparison and statistical analysis of relative expression results in real-time PCR. Nucleic Acids Res. 2002;30(9):e36.

15. Li P, Zhao GQ, Chen TF, et al. Serum miR-21 and miR-155 expression in idiopathic pulmonary fibrosis. J Asthma. 2013;50(9):960-4.

16. Pandit KV, Milosevic J, Kaminski N. MicroRNAs in idiopathic pulmonary fibrosis. Transl Res. 2011;157(4):191-9. 
17. Lin X, Steinberg S, Kandasamy SK, et al. Common miR-590 Variant rs6971711 Present Only in African Americans Reduces miR-590 Biogenesis. PLoS One. 2016;11(5):e0156065.

18. Lesizza P, Prosdocimo G, Martinelli V, et al. Single-Dose Intracardiac Injection of Pro-Regenerative MicroRNAs Improves Cardiac Function After Myocardial Infarction. Circ Res. 2017;120(8):1298-1304.

19. Naghdalipour M, Moradi N, Fadaei R, et al. Alteration of miR-21, miR-433 and miR-590 tissue expression related to the TGF- $\beta$ signaling pathway in ulcerative colitis patients. Arch Physiol Biochem. 2020;15:1-5.

20. Olivieri F, Rippo MR, Monsurrò V, et al. MicroRNAs linking inflamm-aging, cellular senescence and cancer. Ageing Res Rev. 2013;12(4):1056-68.

21. Olivieri F, Albertini MC, Orciani M, et al. DNA damage response (DDR) and senescence: shuttled inflamma-miRNAs on the stage of inflamm-aging. Oncotarget. 2015;6(34):35509-21.

22. Olivieri F, Spazzafumo L, Santini G, et al. Age-related differences in the expression of circulating microRNAs: miR-21 as a new circulating marker of inflammaging. Mech Ageing Dev. 2012;133(1112):675-85.

23. Jung HJ, Suh Y. Circulating miRNAs in ageing and ageing-related diseases. J Genet Genomics. 2014;41(9):465-72.

24. Marts LT, Green DE, Mills ST, et al. MiR-21-Mediated Suppression of Smad7 Induces TGF $\beta 1$ and Can Be Inhibited by Activation of Nrf2 in Alcohol-Treated Lung Fibroblasts. Alcohol Clin Exp Res. 2017;41(11):1875-1885.

25. Yu ZW, Xu YQ, Zhang XJ, et al. Mutual regulation between miR-21 and the TGF $\beta /$ Smad signaling pathway in human bronchial fibroblasts promotes airway remodeling. J Asthma. 2019;56(4):341349.

26. Ekhteraei-Tousi S, Mohammad-Soltani B, Sadeghizadeh M, et al. Inhibitory effect of hsa-miR-590-5p on cardiosphere-derived stem cells differentiation through downregulation of TGFB signaling. $\mathrm{J}$ Cell Biochem. 2015;116(1):179-91.

27. Liu T, Wu Y, Huang T, et al. miR-590 promotes the proliferation of HUMSCs and induces ECM synthesis by targeting Smad7. Oncol Lett. 2017;14(4):3941-3946.

28. Jafarzadeh M, Soltani BM. Hsa-miR-590-5p Interaction with SMAD3 Transcript Supports Its Regulatory Effect on The TGF $\beta$ Signaling Pathway. Cell J. 2016;18(1):7-12.

29. Favreau AJ, Sathyanarayana P. miR-590-5p, miR-219-5p, miR-15b and miR-628-5p are commonly regulated by IL-3, GM-CSF and G-CSF in acute myeloid leukemia. Leuk Res. 2012;36(3):334-41.

30. Derynck R, Budi EH. Specificity, versatility, and control of TGF- $\beta$ family signaling. Sci Signal. 2019;12(570): eaav5183.

\section{Figures}




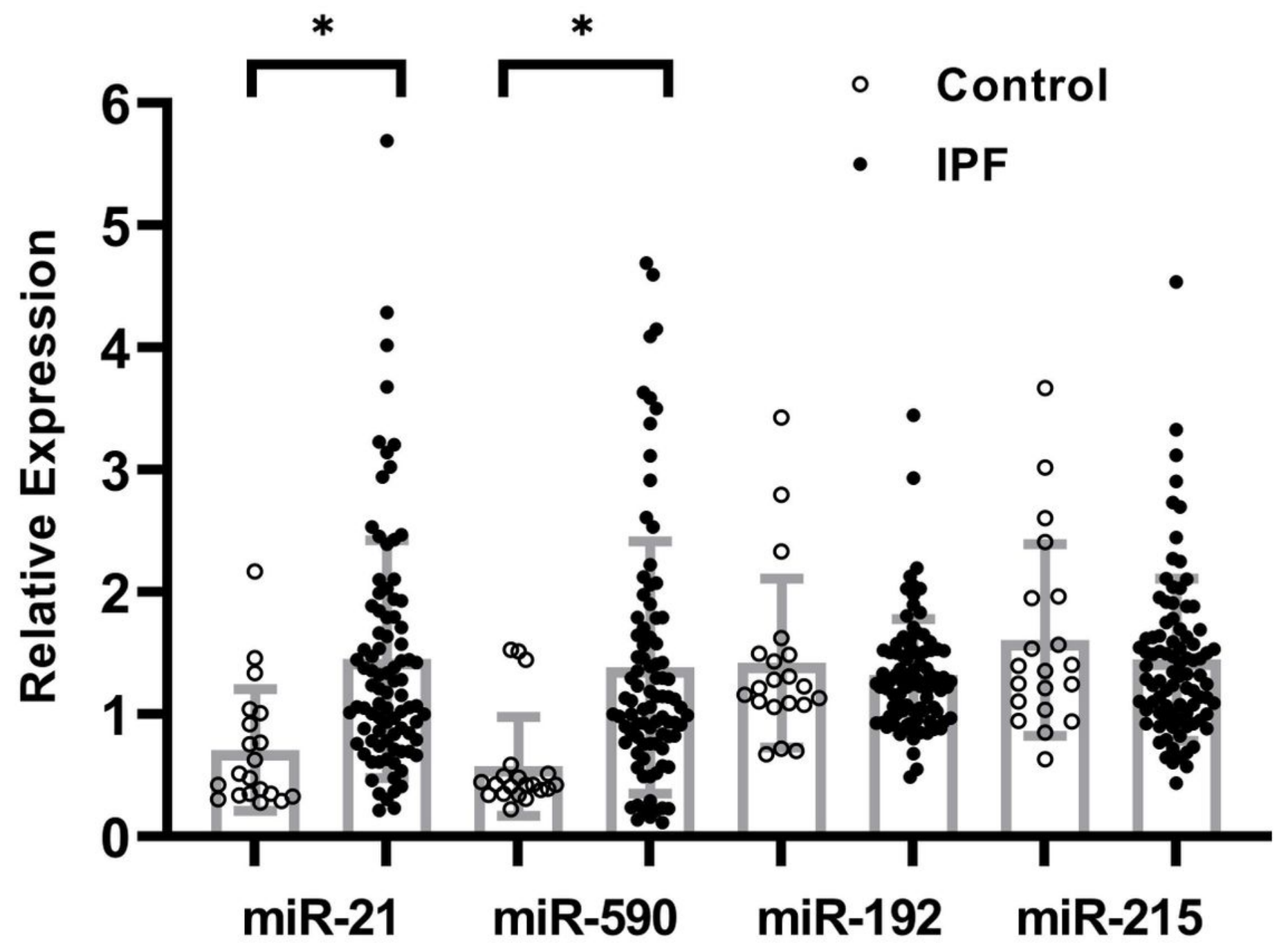

Figure 1

Plasma expression levels of miR-21, miR-590, miR-192, and miR-215 in IPF and control groups. Relative expression level of the target miRNAs was calculated by using miR-16 as a reference. Bars represent means and error lines indicate standard deviation. ${ }^{*} \mathrm{p}<0.0001$. 

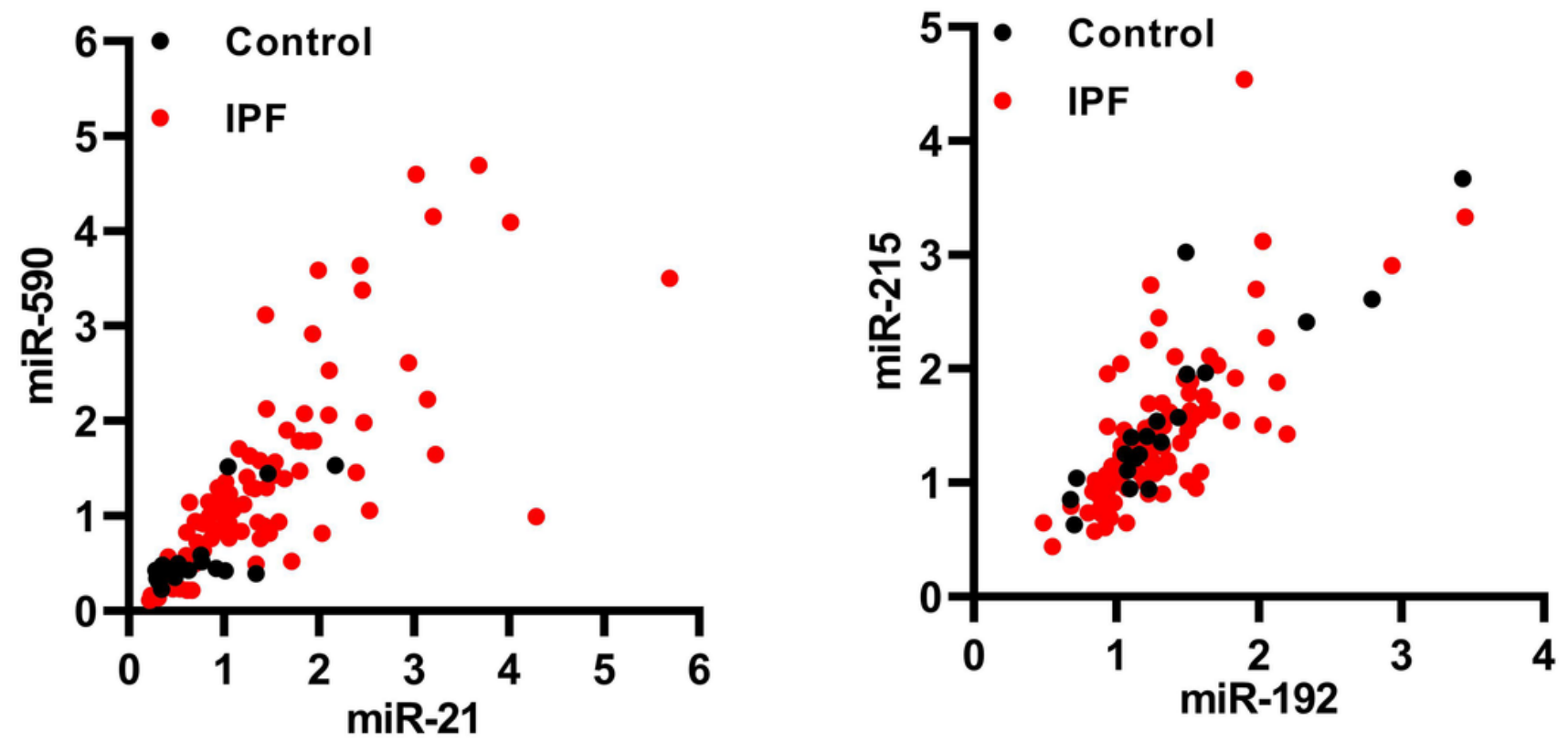

Figure 2

Correlations of miRNAs in the plasma of IPF and control subjects. A, Correlation of miR-21 and miR-590. miR-21\&miR-590 in control group $r=0.68, p=0.0008$ and in IPF group $r=0.79, p<0.0001$. $B$, Correlation of miR-192 and miR-215. miR-192\&miR-215 in control group $r=0.88, p<0.0001$ and in IPF group $r=0.72$, $p<0.0001$.
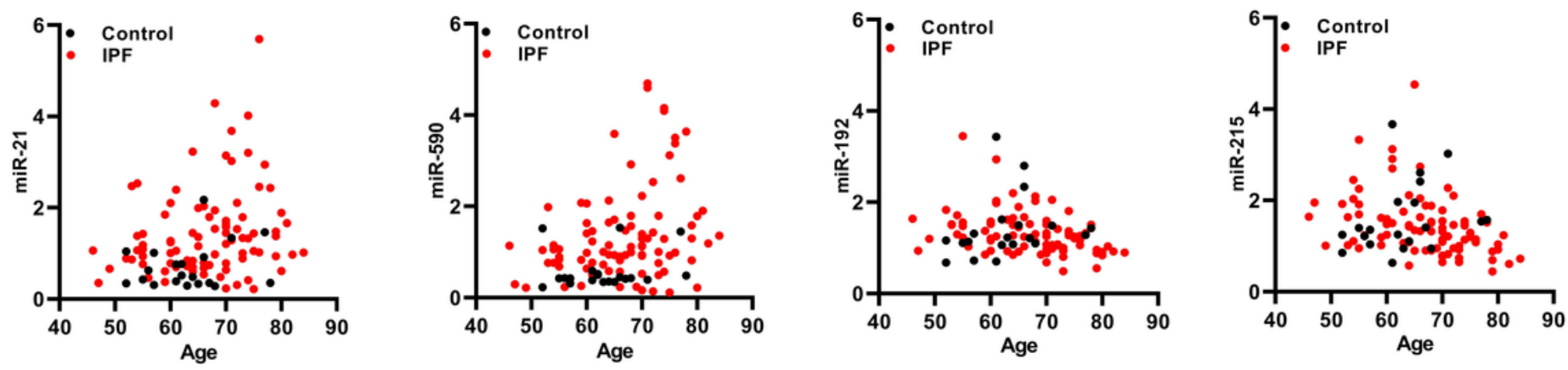

Figure 3

Correlation of age with miRNA in IPF and control groups. Correlation of age (A) with miR-21 (control: $r=0.06, p>0.05$, IPF: $r=0.21, p=0.041$ ), (B) with miR-590 (control: $r=0.17, p>0.05$, IPF: $r=0.28, p=0.007),(C)$ with miR-192 (control: $r=0.42$, $p>0.05$, IPF: $r=-0.38, p=0.0002$ ), with (D) miR-215 (control: $r=0.42, p>0.05$, IPF: $r=-0.44, p<0.0001)$. 

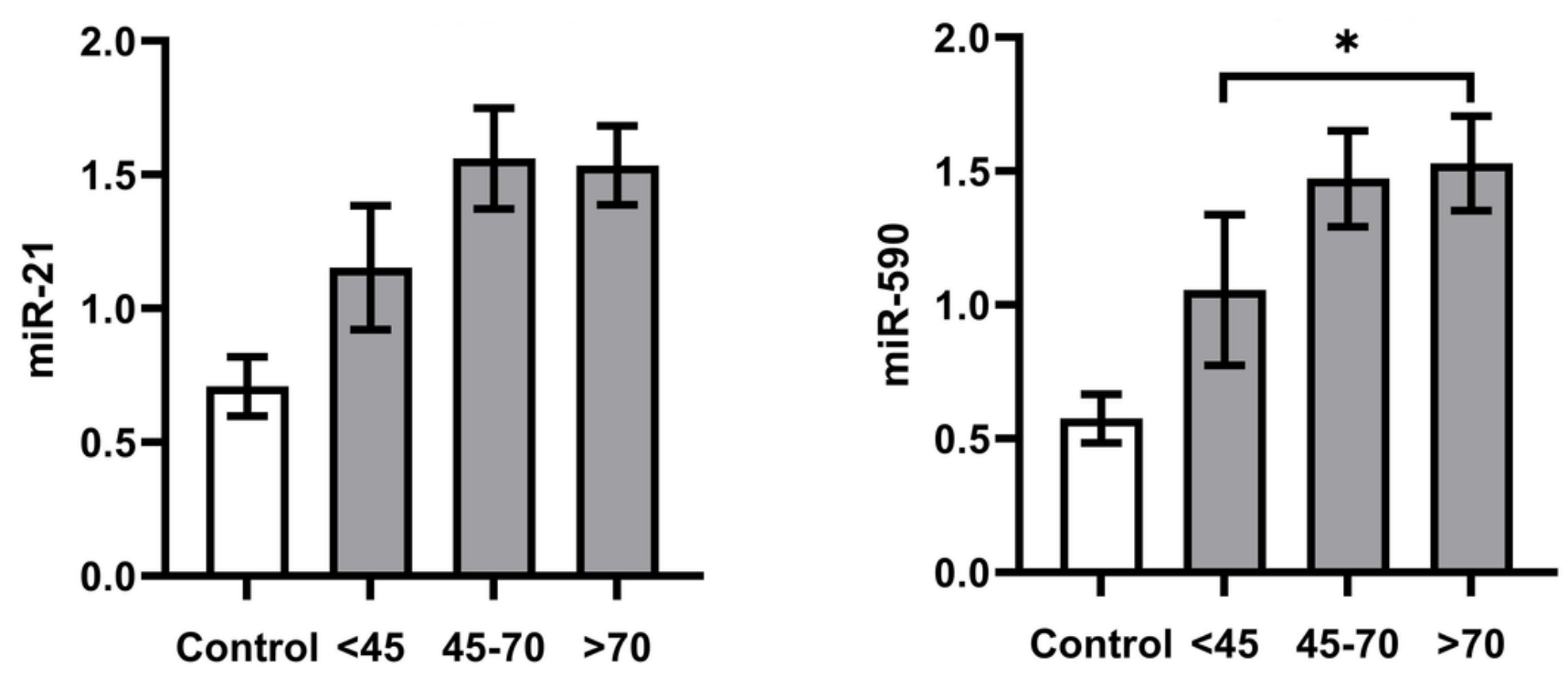

Figure 4

miR-21 and miR-590 levels according to the DLCO subgroups. Plasma (A) miR-21 and (B) miR-590 levels among the subgroups of DLCO $<45 \%, 45-70 \%$, and $>70 \%$ were not significantly different with KruskalWallis test. Bars represent means and error lines indicate SEM. ${ }^{*} \mathrm{p}<0.05$
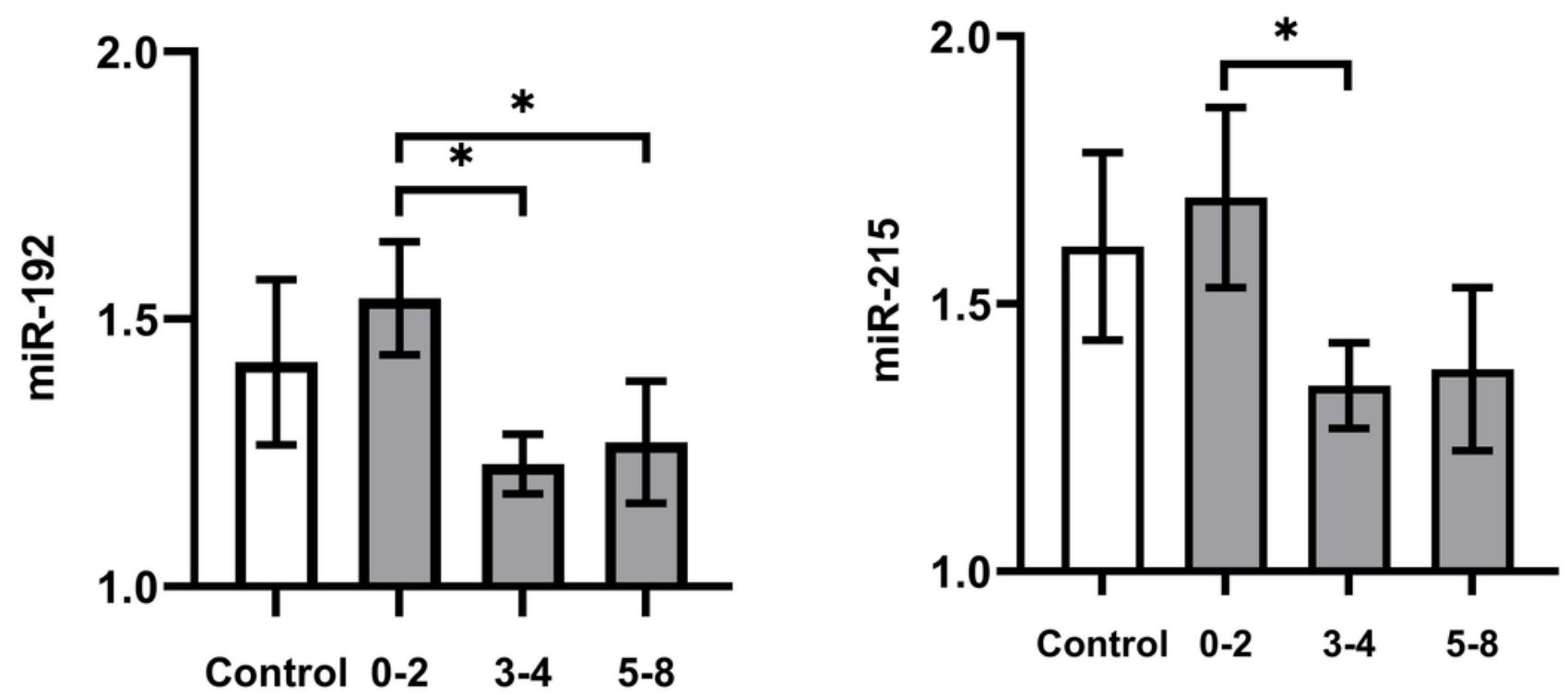

Figure 5

miR-192 and miR-215 levels according to GAP score subgroups. A, miR-192. The plasma miR-192 levels among the subgroups were significantly different with Kruskal-Wallis test $(p=0.010)$. With Dunn`s 
multiple comparison test, GAP 0-2 subgroup was significantly different from other subgroups. B, miR-215. The plasma miR-215 levels among the subgroups were not significantly different with Kruskal-Wallis test. There was a significant difference between miR-215 levels of GAP 0-2 and 3-4 subgroups with MannWhitney U test $(p=0.035)$. Bars represent means and error lines indicate SEM. ${ }^{*} p<0.05$
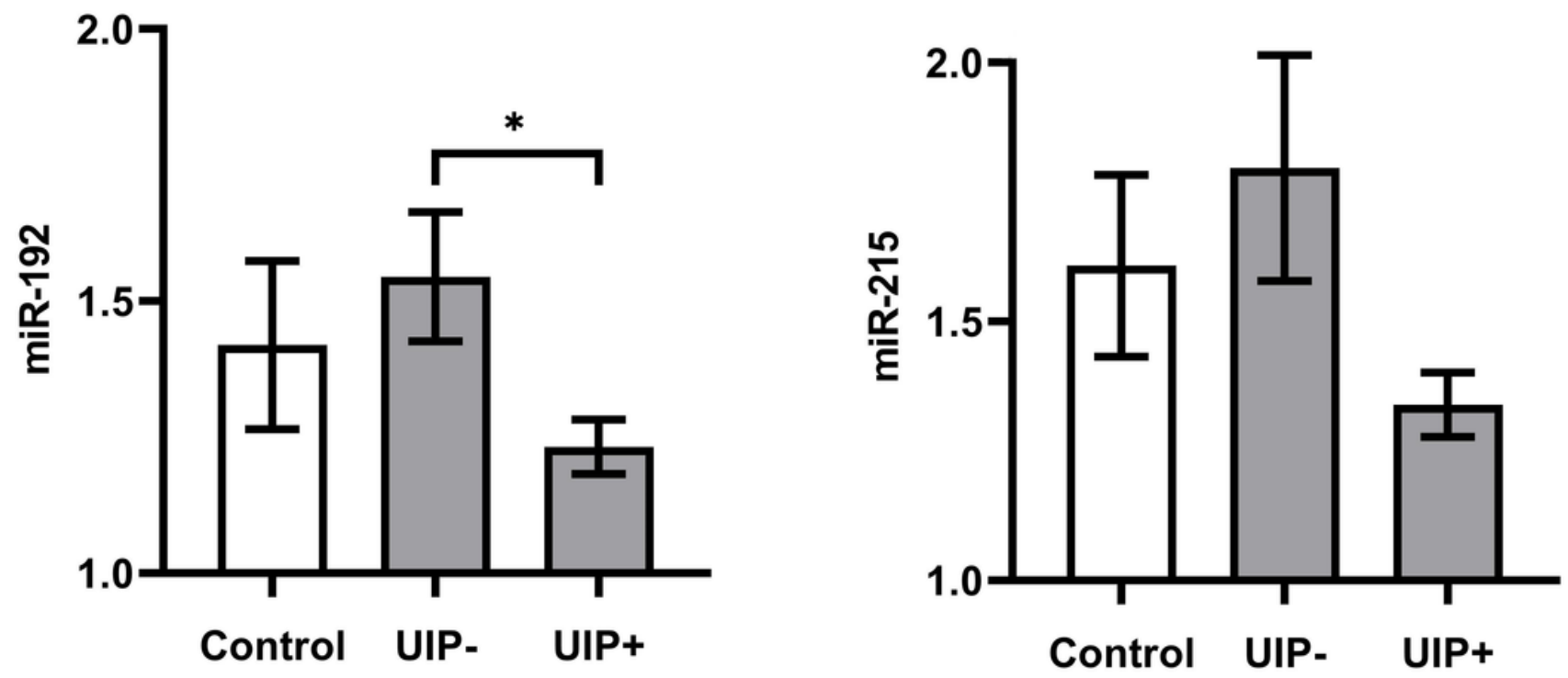

Figure 6

miR-192 and miR-215 levels according to UIP pattern on HRCT. A, Plasma miR-192 levels of the patients with UIP pattern were reduced compared to the patients without it $(p=0.003)$. B, Plasma miR-215 levels were similar between the patients with UIP or without UIP pattern $(p>0.05)$. Bars represent means and error lines indicate SEM. * $p<0.05$

\section{Supplementary Files}

This is a list of supplementary files associated with this preprint. Click to download.

- SupplTables.docx 